\title{
Nanopatterning of Catalyst by Dip Pen Nanolithography (DPN) for Synthesis of Carbon Nanotubes (CNT)
}

\author{
S.-W. KANG ${ }^{1}$, D. BANERJEe ${ }^{1}$, A. B. KAUl ${ }^{2}$, AND K. G. Megerian ${ }^{2}$ \\ ${ }^{1}$ Department of Mechanical Engineering, Texas A\&M University, College Station, Texas \\ ${ }^{2}$ Jet Propulsion Laboratory, California Institute of Technology, Pasadena, California
}

Summary: Carbon nanotubes (CNT) were synthesized on nanopatterned catalysts. The catalyst nanoparticles were obtained using dip pen nanolithographytechnique. Dip pen nanolithography technique was used to deposit $\mathrm{NiCl}_{2}$ nanopatterns with sub$200 \mathrm{~nm}$ feature sizes on a silicon substrate. The deposited features were verified by using lateral force microscopy. The substrate was then placed in a plasma-enhanced chemical vapor deposition instrument to synthesize CNT. The synthesized CNT were characterized using scanning electron microscopy and energy dispersive x-ray spectroscopy. SCANNING 32: 42-48, 2010. (C) 2010 Wiley Periodicals, Inc.

Key words: nanocatalysis, lateral force microscopy (LFM), scanning electron microscope (SEM), spectroscopy, materials characterization

\section{Introduction}

Carbon nanotubes (CNTs) are attractive for their superior material characteristics and tunable properties, such as thermal conductivity, electrical properties, electromagnetic emission characteristics, and mechanical strength (Huang et al. 2007; Salvetat et al. 1999). These characteristics hold promise for applications in high-efficiency energy

Contract grant sponsors: Defense Advanced Projects Agency/ Micro/Nano-Fluidics Fundamental Focus Center (DARPA-MF ${ }^{3}$ ); Office of Naval Research (ONR); Space and Naval Warfare Center Systems Command (SPAWAR-SSC); Department of Energy (DOE).

Address for reprints: D. Banerjee, Mail Stop 3123 TAMU, College Station, TX 77843-3123, Texas, E-mail: dbanerjee@tamu.edu

Received 3 February 2010; Accepted with revision 6 April 2010

DOI $10.1002 /$ sca.20184

Published online 23 April 2010 in Wiley InterScience (www. interscience.wiley.com) storage systems, nanoscale-integrated circuits or sensors, as well as extremely lightweight and strong materials. A single-walled CNT (SWCNT) can be considered to be a single sheet of planar graphite structure that is rolled-up to form a cylindrical structure. These SWCNT materials have tunable metallic or semiconducting properties depending on the crystalline orientation. A multi-walled CNT can be considered to be multiple sheets of graphite planar structures concentrically rolled-up to form a cylindrical structure - having primarily metallic (or mimicking graphitic) properties. Ballistic electron transport in metallic SWCNT makes these materials attractive as channel material in field-effect transistor (FETs), and can provide capabilities not possible with conventional silicon-based FETs (Javey et al. 2004). Previous efforts reported in the literature for the fabrication of SWCNT-FETs required the patterning of well-defined SWCNTs with desired characteristics. These SWCNT patterns required tedious processing steps, such as purification, separation (for desired chirality), and patterning at required locations (Robertson 2007).

Incorporation of CNT materials in commercial nanoelectronics precludes the development of an economical process for synthesizing CNT that is compatible with the CMOS process (Robertson 2007). Such an economical synthesis process should have the ability to: (a) control the chirality of the CNT (metallic or semiconducting); (b) control the patterning or synthesis of the CNT to exact locations (e.g., metallic CNT in vias and semiconducting CNT in the device region); (c) provide synthesis temperatures that are compatible with CMOS process; and (d) provide sufficient number density of CNT at a particular location (e.g., 100 CNT per unit square micron of substrate area). In this study, we describe a process to synthesize CNT that address the above requirements (a)-(d); using a combination of dip pen nanolithography (DPN) and plasma-enhanced chemical vapor deposition (PECVD) techniques. The synthesis process is 
evaluated using materials characterization techniques, such as atomic force microscopy (AFM)/ lateral force microscopy (LFM), scanning electron microscopy (SEM), and energy dispersive $x$-ray spectroscopy (EDS).

\section{Materials and Methods}

Conventional methods for synthesis of CNT, such as carbon arc discharge, laser-vaporization (or laser-ablation technique), chemical vapor deposition (CVD), and electrolysis (Ebbesen and Ajayan 1992; Grobert 2007), do not meet the needs enumerated in criteria (a)-(d) mentioned before. Novel strategies for bulk synthesis of CNT, such as the high-pressure carbon monoxide flow method (Ouellette 2002) result in mixtures of various organic materials, including amorphous carbon, that requires expensive purification procedures. An alternative method for in-situ synthesis using DPN was developed for synthesis of CNT on scanning probe tips (Gargate and Banerjee 2008a,b). In this study, we use a variant of the techniques used by Gargate and Banerjee $(2008 \mathrm{a}, \mathrm{b})$ to synthesize CNT on nanopatterned-catalyst precursors.

CNT are typically synthesized using metal catalysts that form carbide eutectics, such as $\mathrm{Fe}, \mathrm{Pt}, \mathrm{Co}$, $P d$ or $N i$. In this study, we accomplish the nanolithography of precursors to metal catalysts (Nickel Chloride: $\mathrm{NiCl}_{2}$ ). Patterned arrays of $\mathrm{NiCl}_{2}$ with features sizes less than $\sim 200 \mathrm{~nm}$ width and thickness less than $1 \mathrm{~nm}$ were deposited on bare silicon substrate using DPN techniques. CNT synthesis was achieved on these patterns using PECVD.

\section{Nanopatterning of Metal Salts}

DPN enables nanopatterning via the transport of chemical "inks" from the ink-coated scanning probes (the tip or "pen") onto a substrate (the "paper") as depicted in Figure 1, typically using an scanning probe microscope (SPM) platform. As DPN techniques can be used in both contact mode and non-contact mode of SPM, they can prevent any potential damages that can be caused to the samples that are deposited on the surface. Other methods of nanopatterning at this scale of feature sizes (sub-200 nm) suffer from the disadvantage of causing either radiation damage (such as in E-Beam Lithography or Deep UV Lithography) or chemical contamination (such as in DUV/immersion photolithography). More importantly, DPN is capable of depositing the materials precisely to a specifically designated location with nanoscale precision. A primary advantage of DPN is that the same

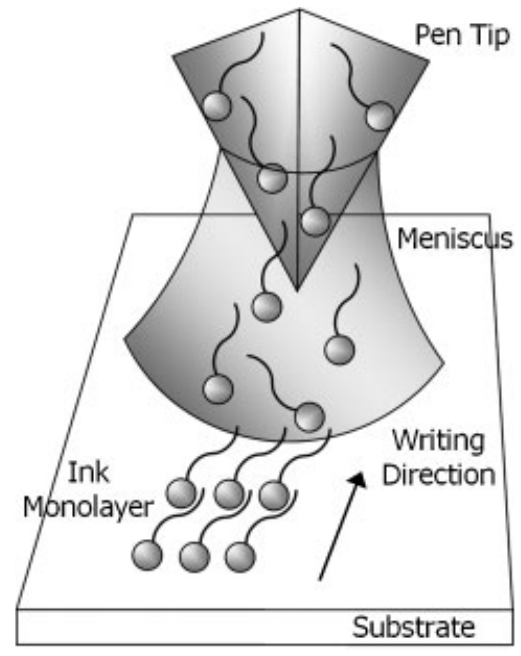

Fig 1. Schematic of DPN "Ink" deposition (Ink molecules are transported onto the substrate through the meniscus bridge formed around tip).

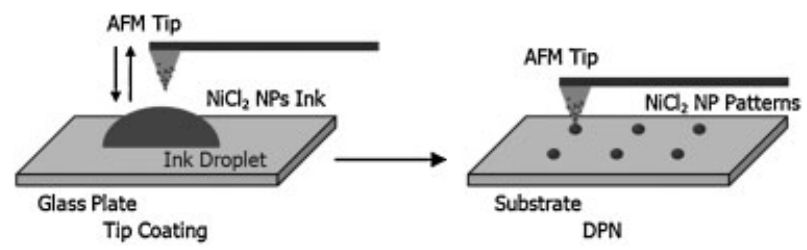

Fig 2. Schematic illustration of the process: AFM tip coating, fabrication of $\mathrm{NiCl}_{2} \mathrm{NP}$ patterns by using DPN (Right-Double dipping procedure/Left-DPN process).

instrument used for patterning (or material deposition) can be used to characterize the deposited samples by using LFM-immediately after the deposition step.

Nanopatterining of the catalyst precursor by DPN is conducted using Nscriptor DPN System (NanoInk, Inc., Skokie, IL) at the materials characterization facility of Texas A\&M University. In general, DPN process includes the following steps: (i) synthesis of ink solution (i.e., aqueous solution of $\mathrm{NiCl}_{2}$ ); (ii) substrate preparation; (iii) tip-coating with ink; (iv) deposition on the substrate (in contact mode or non-contact mode); and (v) verification/characterization of the deposited samples (using LFM). A schematic for this process is shown in Figure 2. The selection of ink materials depends on the surface roughness and the surface diffusivity of the deposited chemical species ( $\mathrm{Li}$ et al. 2008). However, if the surface roughness of a substrate is less than $\sim 3-5 \mathrm{~nm}$, almost any type of material can be used as ink for deposition on the substrate - provided the material can be coated on the scanning probe tip or "pen" (Hong et al. 1999; Piner et al. 1999; Schwartz 2002; Weeks and Vaughn 
2005). In this study, $\mathrm{NiCl}_{2}$ is dissolved to form $0.008 \mathrm{M}$ aqueous solutions, by mixing of $10 \mathrm{mg}$ of $\mathrm{NiCl}_{2}$ powder and $10 \mathrm{~mL}$ of DI water using an ultara-sonicator for $30 \mathrm{~min}$. This results in a clear solution of $\mathrm{NiCl}_{2}$ as shown in Figure 3(B)

Sindex ${ }^{\mathrm{TM}}$ chip (Bare $\mathrm{Si}$ substrate; purchased from Bioforce Nanosciences, Ames, IA) was used as the substrate for the deposition of the catalyst precursor. A Sindex chip consists of $4 \times 4 \mathrm{~mm}$ silicon substrate containing topographically defined mesa pads that are arrayed within an alphanumeric indexing system. There are three patterned regions on each chip: 50, 100, and $200 \mu \mathrm{m}$ square regions as shown in Figure 3(A). Before conducting the DPN experiment, the substrate is rinsed with acetone and deionized water and dried in air. We applied the "double-dipping procedure" for preparing the "pen" by coating the tip with the desired "ink" (Gargate and Banerjee 2008a,b). The pen is prepared by using silicon nitride probes (Model: Single Probes of Type A with spring constant of $0.041 \mathrm{~N} / \mathrm{m}$, purchased from NanoInk, Inc.). There are one rectangular and triangular tips of different stiffness in this pen. Among these tips, we use the tip of rectangular shape as summarized in Table I. As shown in Figure 2, the probe can be coated by immersing the tip in a droplet of ink solution for 1-2 min and air-drying for $30 \mathrm{sec}$. This process is repeated twice for enhancing the effect of coating (hence the name "Double Dipping Procedure"). Because the backside of the cantilever does not contact the ink material and remains clean in this method, it can prevent the attenuation of the AFM feedback signal (Li et al. 2008). After dipping process, the "pen" is
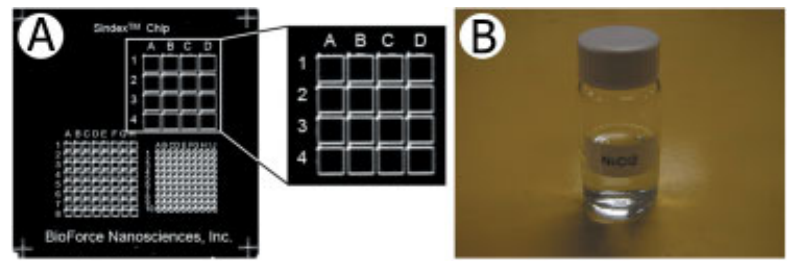

Fig 3. Image of substrate and ink solution: (A) Sindex ${ }^{\mathrm{TM}}$ chip (DPN experiment is conducted in the enlarged area) (B) Liquid $\mathrm{NiCl}_{2}$.

TABLE I DPN pen probe specification (Nanoink, Inc.-A Type)

\begin{tabular}{|c|c|c|}
\hline$[\overline{1}$ & & A-1 rectangular \\
\hline$\rightarrow+$ & Length $(l)(\mu \mathrm{m})$ & 200 \\
\hline \multirow{4}{*}{$A$} & Width $(w)(\mu \mathrm{m})$ & 45 \\
\hline & Thickness $(\mu \mathrm{m})$ & 0.6 \\
\hline & Frequency $(\mathrm{kHz})$ & 27 \\
\hline & Stiffness $(\mathrm{N} / \mathrm{m})$ & 0.041 \\
\hline
\end{tabular}

ready for deposition of "ink" on the desired substrate.

It is widely believed that the ink deposition in DPN occurs due to the formation of a "chemical bridge" by means of a nanodroplet of water on the probe tip from the ambient humidity. The nanodroplet is formed by condensation of water vapor on the probe tip - from ambient humidity - due to the high surface energy of the tip (which has a tip radius of $\sim 10-30 \mathrm{~nm}$ ), when brought in close proximity to a flat substrate due to confinement of the vapor molecules within a small space (Banerjee 2007; Schwartz 2002). The ink on the pen then dissolves in the water droplet formed at the tip and then transported to the surface in contact with the water droplet ("chemical bridge"). The ink molecules that spread on the substrate by a surface-diffusionmediated process that depends on the concentration gradient. This surface diffusion can be impeded severely if the surface roughness exceeds $3-5 \mathrm{~nm}$. As deposition process in DPN is a diffusion-mediated process across a surface concentration gradient, larger dots (or patterns) can be obtained by increasing the dwell time (or touching time) of the tip to the substrate for a particular temperature. However, it should be noted that this is not an universal phenomenon, because such a behavior is not observed for inks consisting of large molecules (such as proteins), where a stepwise behavior is observed for patterned features as a function of dwell time, which is probably due to the non-linear nature of molecular transport that is involved during surface diffusion of large molecules. Figure 4 shows that in the case of patterned lines (for inks composed of materials with small molecules), increasing the speed of writing (or the speed of moving the tip across a surface) causes thinner line patterns to be obtained. At sufficiently high-tip speeds (or above a threshold speed), the nanodroplet is believed to be disrupted, causing the tip to become "dry". Hence, the same tip can be used to scan the deposited material using LFM technique, provided the scanning speed is above this threshold value.

The ambient relative humidity around the pen tip can have a significant effect on the writing process (Banerjee 2007; Weeks and Vaughn 2005). Higher relative humidity favors the formation of a larger water meniscus (or water droplet) around the tip and helps with the formation of larger surface patterns. Thus, in our experiment, the ambient relative humidity is controlled to be at least $60 \%$ or greater at room temperature. As experimental parameters we mentioned above (including the process temperature) simultaneously affect the size of the nanopatterns, the effect for one parameter can be estimated consistently by controlling all other parameters to a constant value. 
A

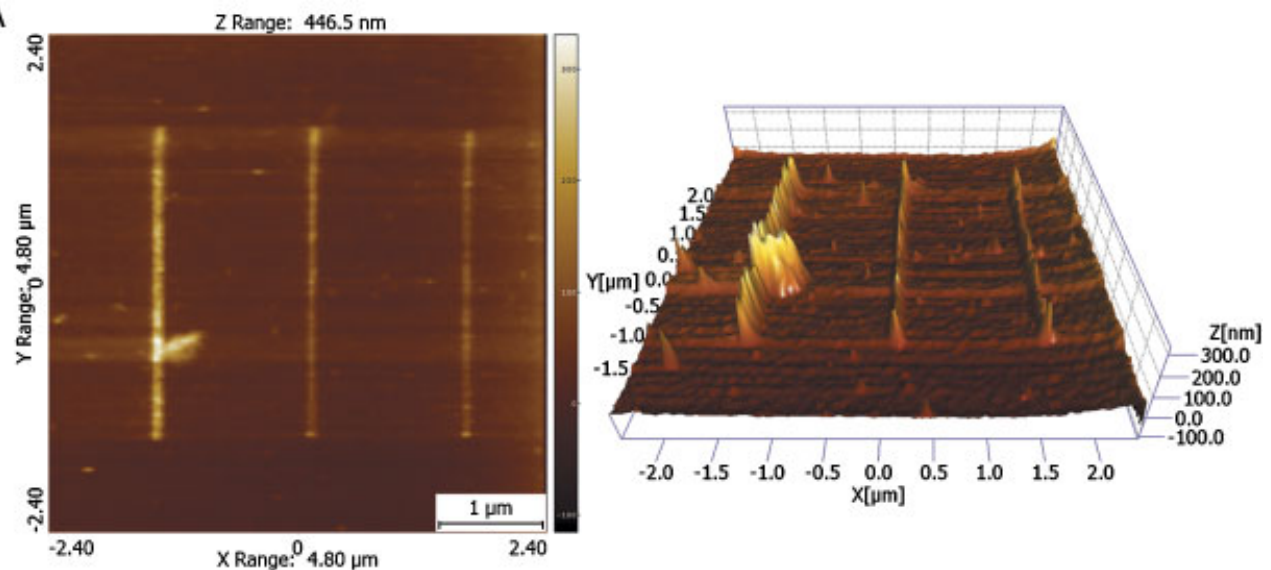

B

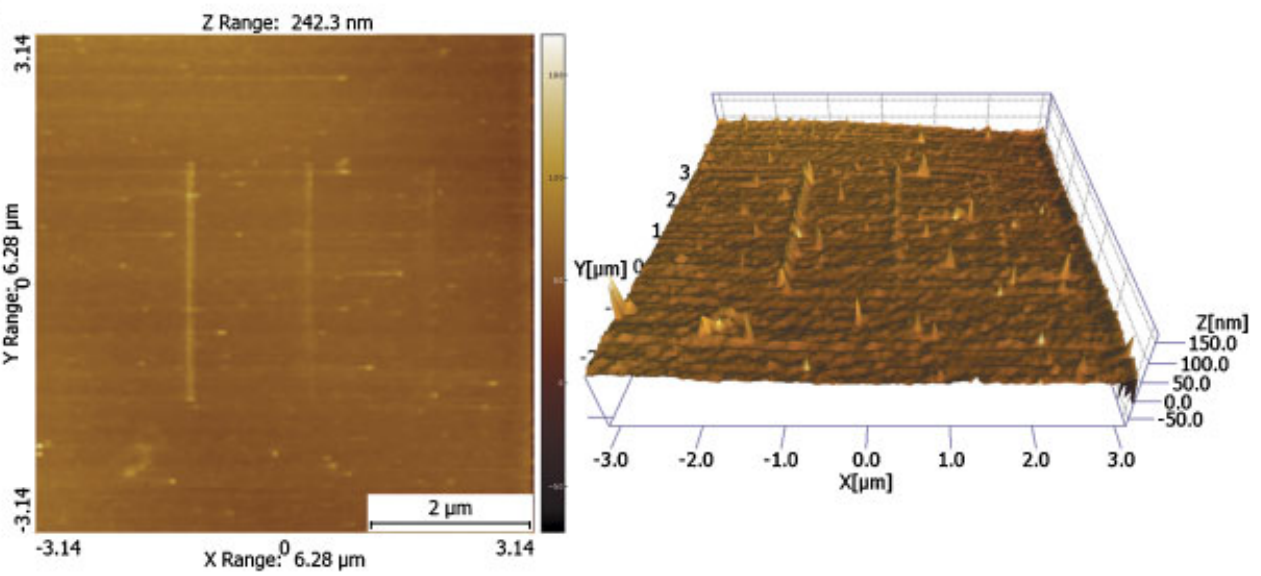

Fig 4. Lateral force microscopy (LFM) Image of $\mathrm{NiCl}_{2}$ deposited by DPN on Sindex ${ }^{\mathrm{TM}}$ chip: (A) Writing speed ( $\left.\mu \mathrm{m} / \mathrm{s}\right): 0.1,0.2$, 0.4 , Scan size: $4.80 \mu \mathrm{m}$, Scan speed: $3 \mathrm{~Hz}(\mathbf{B})$ Writing speed $(\mu \mathrm{m} / \mathrm{s}): 0.05,0.1,0.15$, Scan size: $6.30 \mu \mathrm{m}$, Scan speed: $3 \mathrm{~Hz}$.

\section{Synthesis of CNTs (Description of PECVD Process)}

A technique which has emerged in recent years to ensure excellent vertical tube alignment is PECVD (Ren et al. 1998), where the inherent electric field in the plasma allows tube growth in a direction parallel to the field. Analysis of crystallinity of individual tubes obtained from typical PECVD process reveals graphitic structures, where the graphene layers are inclined to the central axis (Nolan et al. 1998) and the structures are commonly referred to as carbon nanofibers (CNFs); however, control over their physical orientation with the plasma is excellent. Various plasma sources have been employed for tube or CNF growth, such as microwave, inductively coupled plasma, de and de with hot filament; a comprehensive review of the PECVD technique for tube synthesis is provided by Melechko et al. (2005).

Besides the ability to direct growth orientation, another important figure of merit for many applications is the precise control over tube placement. There have been many reports on the PECVD growth of multiple tubes formed at controlled locations using positive tone novalac/diazoquinonebased or negative tone rubber/azide resists. When only single tubes are desired where the catalyst size has to be controlled below a few hundred nanometers, such resist systems show excessive absorption in the deep-UV region, and are inadequate for deep-submicron feature size resolution. To date, e-beam lithography has been the main technique implemented for catalyst definition for the realization of arrays of individual, free-standing, vertically aligned CNTs or CNFs using PECVD (Teo et al. 2003). However, e-beam lithography is slow and expensive, and ultimately this limits the transition of nanoscale devices from the laboratory into commercial production.

A schematic of our load-lock-based PECVD growth chamber is provided elsewhere, where a base pressure of $\sim 5 \times 10^{-8}$ Torr was typically achieved (Kaul et al. 2009). The as-prepared sample was placed on a 3-inch Mo ring and was transported from the load lock to the main chamber. The assembly was lowered onto a holder, with a resistive 

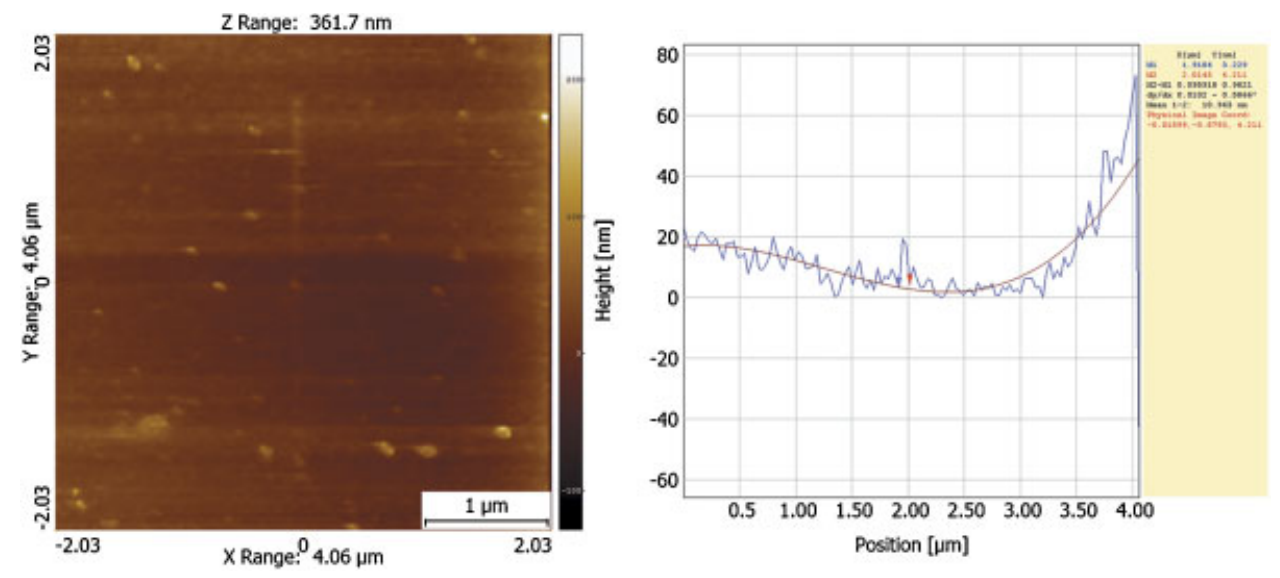

Fig 5. Line analysis of $\mathrm{NiCl}_{2}$ deposited by DPN on Sindex ${ }^{\mathrm{TM}}$ chip (Writing speed is $0.28 \mu \mathrm{m} / \mathrm{s}$ and its feature size is $\sim 96 \pm 1 \mathrm{~nm}$ ).

heater beneath, where the temperature was ramped with an Athena Controls, Inc., Plymouth Meeting, PA (Series 6080) temperature controller. The mount on which the Mo ring/wafer rested, served as the cathode for dc plasma operation. A circular metal disk approximately $\sim 8 \mathrm{~mm}$ above the cathode was electrically isolated and served as the anode. The flow of gases into the chamber was controlled by mass flow controllers (Sierra Instruments-6514) and the pressure during growth was measured with an MKS (PDR-C-2C) capacitance manometer, and controlled by an exhaust valve controller (Type 252A). The plasma was powered by an Advanced Energy, Inc. (MDX Magnetron Drive) dc power supply.

The substrate with the $\mathrm{NiCl}_{2}$ catalyst precursor (deposited using DPN) was exposed to a pretreatment step for $1 \mathrm{~min}$ before CNT growth, where a lowpower hydrogen plasma ( $60 \mathrm{~W}, 5$ Torr) reduced the surface oxide on the $\mathrm{Ni}$ at $700^{\circ} \mathrm{C}$. After pretreatment, the chamber was pumped out until the pressure was restored to $\sim 2 \times 10^{-6}$ Torr, after which point highpurity acetylene $\left(\mathrm{C}_{2} \mathrm{H}_{2}\right)$ and ammonia $\left(\mathrm{NH}_{3}\right)$ were introduced, which served as the carbon feedstock and diluent gas, respectively. When the pressure in the chamber had stabilized to $\sim 5$ Torr, the dc discharge was ignited, and growth was carried out for $5 \mathrm{~min}$. After completion, the samples were immediately transferred into the load lock for cooling and imaged using the SEM (JEOL-6700F).

\section{Results}

Verification of the feature size for the deposited catalyst precursors is accomplished using LFM. Figure 4 shows LFM images of line patterns of $\mathrm{NiCl}_{2}$ that were generated by the DPN process on Sindex ${ }^{\mathrm{TM}}$ chip. Figure 5 shows the line analysis performed using SPIP ${ }^{\mathrm{TM}}$ software tools (Scanning Probe Image Processor: Image Metrology Co.,

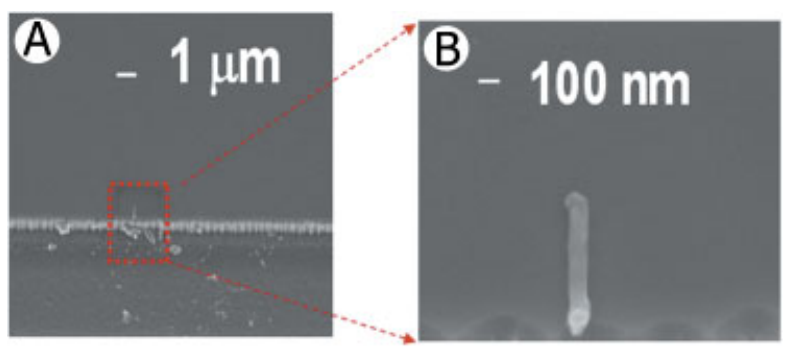

Fig 6. (A) Left image is a low magnification image of a single vertically oriented tube. (B) A high magnification SEM image of the same tube. Tilt angle in the SEM is 30 degrees.

Hørsholm, Denmark) for measuring the feature sizes patterned by using DPN. The line patterns shown in Figure 4 were measured to be in the range of 100 200 nm width. When we analyze the features based on line analysis, the maximum writing speed used to obtain the line patterns by DPN in this experiment is $0.28 \mu \mathrm{m} / \mathrm{sec}$, and the width of the minimum feature size is $\sim 96 \pm 1 \mathrm{~nm}$, as shown in Figure 5.

After the DPN catalyst was defined using the described procedures, the sample was placed in the PECVD furnace to explore the likelihood of carbonnanotube growth with such a catalyst. On SEM analysis of the sample after PECVD growth, high aspect ratio features were observed on the chip, as shown by the SEM images in Figure 6. Depicted in Figure 6(A) is a low magnification SEM image of such a feature. The high-magnification SEM image in Figure 6(B) is suggestive of a tubular, high aspect ratio nanostructure, similar to what is observed with the synthesis of CNTs, or potentially nanofibers that are typically seen with PECVD. The SEM images were taken at a tilt angle of $30^{\circ}$, and indicate a uniform tubular diameter of $\sim 80 \mathrm{~nm}$, with a length of $\sim 1 \mu \mathrm{m}$, which is in the range for the growth rate that is expected with the PECVD process.

We then proceeded to do EDS of the various regions within the nanostructure shown in Figure 6, 
A

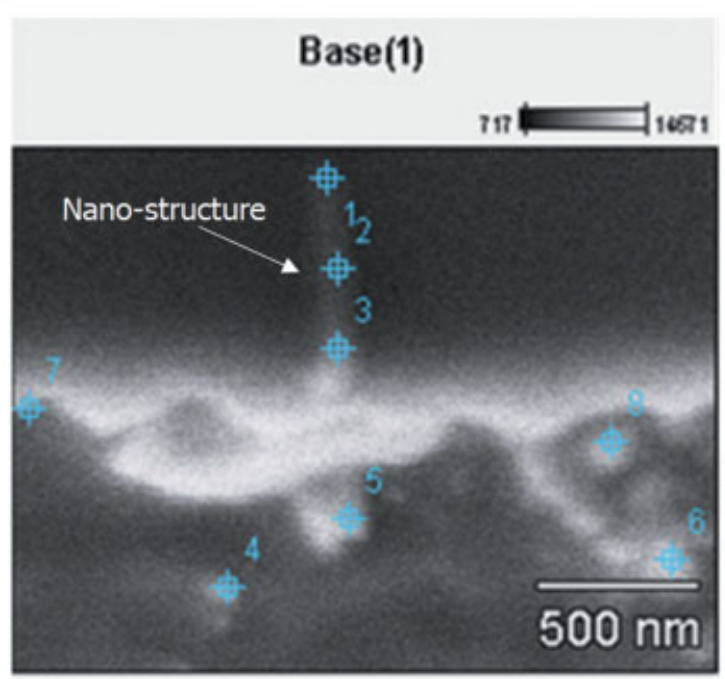

B

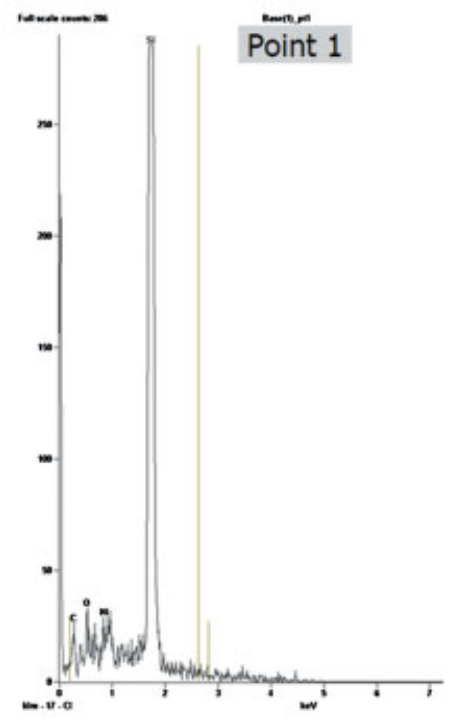

C

D

E

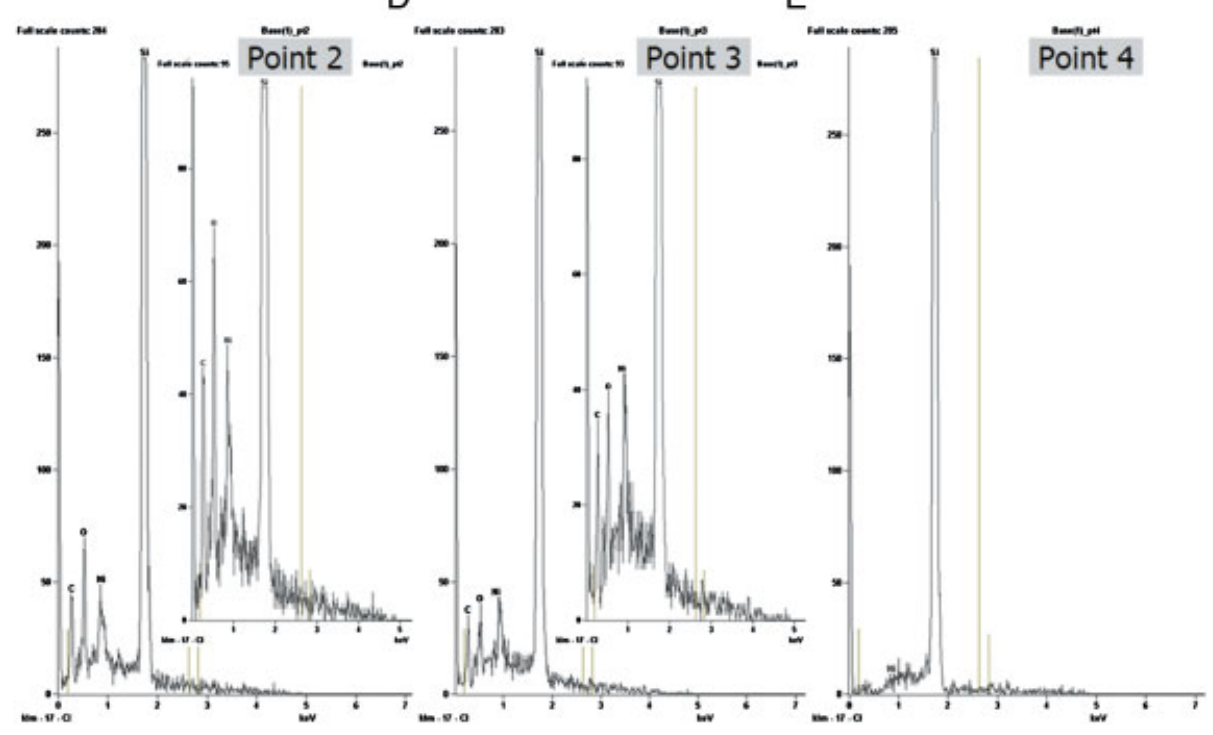

Fig 7. (A) Image of the nanostructure from Figure 6(B), indicating regions (1-8), where EDS spectra were obtained. Regions 1, 2 , and 3 indicate are near the top, within the body and near the base of the tube. (B) EDS spectra taken in Region 1 near the top of the tube suggesting presence of carbon, nickel and oxygen. Similarly, in Region 2 and 3, the EDS spectra in (C) and (D) show the intensity of the carbon and oxygen are enhanced. (E) In regions away from the tube, for example, Region 4, the spectra indicates the absence of carbon, suggesting that the nanostructure in A is composed of a high-aspect ratio carbon nanostructure that was synthesized using a combination of DPN for catalyst definition and dc PECVD for the tube synthesis.

to gather some insight into the chemical composition at various regions outlined by the points $(1-8)$ in the image of Figure 7(A). From this preliminary data, we see that the carbon signal is enhanced within the body and tip of the nanostructure, for example as indicated by the EDS spectra in Figure 7(B-D) that are taken for Region 1, 2, and 3, respectively. In the regions that are outside the proximity of the tube, for example, Region 4, the strength of the carbon peak diminishes, as indicated by the EDS spectra in Figure 7(E). The spectra in Figure 7(B-D) also indicate the presence of oxygen, suggesting some oxidation is occurring at the surface of the nanostructure at the high growth temperature. In addition, the Si peak appears to be from the background as the spot size on our beam was wider than the diameter of the tube. In addition, although more data is necessary, the Ni peak appears to diminish from the top (Fig. 7(B)) to the bottom of the tube (Fig. 7(D)), suggesting that a base growth mechanism may predominate with the combination of DPN catalyst and PECVD, which is unlike previous observations with evaporated $\mathrm{Ni}$ catalyst, where a tip-growth mechanism is usually observed.

Although more work is necessary to characterize factors, such as yield, and crystallographic structure of the synthesized structures, but from our 
preliminary results, it appears that the synthesized nanostructures appear to be carbon-rich and with their tubular, high-aspect ratio morphologies they appear to be suggestive of carbon nanostructures, such as CNT or CNFs. This appears to be the first report where DPN catalyst has been used in conjunction with dc PECVD for nanotube synthesis, and is a resist-free processing approach for catalyst definition for the realization of aligned carbon nanostructures. Such an integration of DPN with PECVD has the potential to enhance flexibility for realizing architectures not otherwise possible with conventional resist-based fabrication techniques, for enabling a wide variety of applications in electronics, biology, and sensing.

\section{Conclusion}

This study demonstrates the feasibility of synthesizing CNT by using pre-patterned features consisting of catalyst precursors. The precursors are expected to decompose into catalyst metal nanoparticles with sub- $100 \mathrm{~nm}$ feature sizes which serve as the catalyst "seeds" for growth by alternate sequence of formation of metal carbides and precipitation of supersaturated carbon in the form of a tube around the catalyst nanoparticles/carbides, resulting in the formation of CNT structures. It can be expected that the size of the patterned features control the size of the catalysts nanoparticles that are obtained in the process, which in turn control the size of the CNT yielded by the technique. Using feature sizes ranging from approximately 90 to $200 \mathrm{~nm}$ for the catalyst precursors, CNT were synthesized with controlled location, properties, and number density on a substrate.

\section{Acknowledgements}

During the execution of this study, S.-W. Kang was supported through the Defense Advanced Projects Agency/Micro/Nano-Fluidics Fundamental Focus Center (DARPA-MF ${ }^{3}$ ). During the execution of this study, D. Banerjee was supported through the American Society for Engineering Education (ASEE)-Office of Naval Research (ONR)/Summer Faculty Fellowship Program (SFFP) at the Space and Naval Warfare Center Systems Command (SPAWAR- SSC). D. Banerjee also acknowledges the support of ONR through the Thermal Management Program organized by Dr. Mark Spector. In addition, during the execution of this study, D. Banerjee was partially supported through the Concentrated Solar Power (CSP) program from the Department of Energy (DOE).

\section{References}

Banerjee D: BioMEMS and biomedical nanotechnology-III-1. In Dip Pen Technologies for Bio-molecular Devices, Vol. 1, 1st ed., (Ferrari, M, Ed.), Springer, Berlin (2007).

Ebbesen T, Ajayan P: Large-scale synthesis of carbon nanotubes. Nature 358, 220-222 (1992).

Gargate RV, Banerjee D: Room temperature synthesis of carbon nanotubes using dip pen nanolithography (DPN). Proceedings of the SPIE Micro (MEMS) and Nanotechnologies for Defense and Security Symposium, Orlando, FL, 6959, 69590K-69590K-7 (2008a).

Gargate RV, Banerjee D: In situ synthesis of carbon nanotubes on heated scanning probes using dip-pen techniques. Scanning 30, 151-158 (2008b).

Grobert N: Carbon nanotubes-becoming clean. Mater Today 10, 1-2, 28-35 (2007).

Hong S, Zhu J, Mirkin CA: Multiple ink nanolithography: toward a multiple-pen nano-plotter. Science $\mathbf{2 8 6}$, 523-525 (1999).

Huang L, Jia Z, O'Brien S: Orientated assembly of singlewalled carbon nanotubes and applications. $J$ Mater Chem 17, 3863-3874 (2007).

Javey A, Guo J, Farmer DB, Wang Q, Yenilmez E, et al.: Self-aligned ballistic molecular transistors and electrically parallel nanotube arrays. Nano Letters 4, 1319-1322 (2004).

Kaul AB, Megerian KG, von Allmen P, Baron RL: Single, aligned carbon nanotubes in $3 \mathrm{D}$ nanoscale architectures enabled by top-down and bottom-up manufacturable processes. Nanotechnology 20, 075303 (2009).

Li B, Goh CF, Zhou X, Lu G, Tantang H, et al.: Patterning colloidal metal nanoparticles for controlled growth of carbon nanotubes. Adv Mater 20, 4873-4878 (2008).

Melechko AV, Merkulov VI, McKnight TE, Guillorn MA, Klein KL, et al.: Vertically aligned carbon nanofibers and related structures: controlled synthesis and directed assembly. J Appl Phys 97, 041301 (2005).

Nolan D, Lynch DC, Cutler AH: Carbon deposition and hydrocarbon formation on group VIII metal catalysts. J Phys Chem B 102, 4165 (1998).

Ouellette J: Building the nanofuture with carbon tubes. Ind Phys, December 18-21 (2002).

Piner RD, Zhu J, Xu F, Hong S, Mirkin CA: Dip-pen nanolithography. Science 283, 661-663 (1999).

Ren ZF, Huang ZP, Xu JW, Wang JH, Bush P, et al.: Synthesis of large arrays of well-aligned carbon nanotubes on glass. Science 282, 1105 (1998).

Robertson J: Growth of nanotubes for electronics. Mater Today 10, 1-2, 36-43 (2007).

Salvetat J-P, Bonard J-M, Thomson NH, Kulik AJ, Forró L, et al.: Mechanical properties of carbon nanotubes. Appl Phys 69, 255-260 (1999).

Schwartz PV: Molecular transport from an atomic force microscope tip: a comparative study of dip-pen nanolithography. Langmuir 18, 4041-4046 (2002).

Teo KBK, Lee SB, Chhowalla M, Semet V, Binh VT, et al:: Plasma enhanced chemical vapour deposition carbon nanotubes/nanofibres - how uniform do they grow? Nanotechnology 14, 204 (2003).

Weeks BL, Vaughn MW: Direct imaging of meniscus formation in atomic force microscopy using environmental scanning electron microscopy. Langmuir 21, 8096-8098 (2005). 